\title{
Performance Analysis of IEEE 802.11 DCF in Binary Symmetric Channels
}

\author{
Yu Zheng $\dagger$, Kejie Lu*, Dapeng Wu†, and Yuguang Fang $\dagger$ \\ $\dagger$ Department of Electrical and Computer Engineering \\ University of Florida, Gainesville, FL 32611, USA. \\ * Department of Electrical and Computer Engineering \\ University of Puerto Rico at Mayagüez, Mayagüez, PR 00681, USA.
}

\begin{abstract}
IEEE 802.11 is the most important standard for wireless local area networks (WLANs). In IEEE 802.11, the fundamental medium access control (MAC) scheme is distributed coordination function (DCF), whose performance has been studied analytically in the literature. However, to the best of the authors' knowledge, there is no accurate model that takes into account both the incoming traffic loads and the effect of bit transmission errors, which, in addition to collision, can also result in unsuccessful packet delivery. In this paper, we address this issue and provide a new analytical model to evaluate the performance of DCF in binary symmetric channels (BSCs). In our study, we consider the impact of different factors together, including the binary exponential backoff mechanism in DCF, various incoming traffic loads, distribution of incoming packet size, queueing system at the MAC layer, and the packet transmission errors, which has never been done before. Extensive simulation and analysis results show that our analytical model can accurately predict the delay and throughput performance of IEEE 802.11 DCF under different traffic and transmission error conditions.
\end{abstract}

\section{INTRODUCTION}

Wireless local area networks (WLANs) have been widely deployed in recent years. In WLANs, the most important standard is IEEE 802.11 [1], in which the fundamental medium access control (MAC) scheme is distributed coordination function (DCF), which is a carrier sense multiple access with collision avoidance (CSMA/CA) protocol. To better understand the performance of WLANs, a critical challenge is how to analyze IEEE 802.11 DCF. This topic has attracted a lot of research interests in the literature.

To analyze the performance of IEEE 802.11 DCF, most early studies consider a simplified scenario, where every node in the network has a packet to transmit at any time, known as the saturated condition. In addition, we note that most works also ignore the impact of the queue at the MAC layer. Recently, the performance of DCF under unsaturated traffic condition and MAC layer queue has been studied in [2]-[4].

The model in [2] is based on a G/G/1 queue. Due to computational complexity, this model depends on several approximated parameters, such as the probability that a node has no packet to transmit. Consequently, the analysis results have a large derivation in comparison to the simulation results. Moreover, this model is not suitable for high traffic load condition since the queue size is assumed to be infinite, which may not be valid in practice.
The finite capacity of the queue is studied in [3] and [4]. The models in [3] and [4] are common in that both of the analyses are based on the $\mathrm{M} / \mathrm{G} / 1 / \mathrm{K}$ queue. In addition, both of them require iterative algorithms. This is because, to solve the $\mathrm{M} / \mathrm{G} / 1 / \mathrm{K}$ model, the service time distribution is required, while to calculate the service time distribution, a required parameter is the probability that a node has no packet to transmit, which can be achieved by solving the $\mathrm{M} / \mathrm{G} / 1 / \mathrm{K}$ model. The main difference of these two models is how to calculate the service time distribution. In [3], Ozdemir and McDonald proposed to use the Markov modulated general independent model. In contrast, Zhai, Kwon and Fang [4] used a transfer-function approach to calculate the service time distribution directly. In [5], we developed a more accurate and tractable algorithm using a similar technique as [4].

From the discussion above, we note that an important and realistic condition - packet transmission error, has not been addressed adequately. The only study that takes the packet transmission errors into account is [6], where the analysis, however, is based on the saturated condition. To the best of the authors' knowledge, there is no analytical model that considers the packet transmission error conditions in the unsaturated performance analysis for IEEE 802.11 DCF.

In this paper, we provide a new analytical model to evaluate the performance of DCF in binary symmetric channels, which have been widely used for evaluating the performance of MAC protocols in the literature. In this study, we consider the impact of different factors together, including the binary exponential backoff mechanism in DCF, various incoming traffic loads, distribution of incoming packet size, queueing system at the MAC layer, and the packet transmission errors, which has never been done before. Extensive simulation and analysis results show that our analytical model can accurately predict the delay and throughput performance of IEEE 802.11 DCF under different traffic and packet transmission error conditions.

The rest of the paper is organized as the follows. In Section II, we will focus on the analytical model for the unsaturated performance of DCF in a realistic WLAN. Simulation and numerical results will be shown in Section III. Finally, Section IV concludes the paper. 


\section{Analytical Model for IEEE 802.11 DCF}

In this section, we present an analytical model to evaluate the performance of IEEE 802.11 DCF with packet transmission errors. In this model, we also take into consideration a number of realistic conditions, including incoming traffic loads, packet size distribution, and the queueing behavior. Similar to [3] and [4], in our study we will decompose the MAC into two subsystems: the queueing subsystem that takes care of the queueing behavior based on the M/G/1/K model; and the service subsystem that characterizes the service time distribution.

The rest of this section is organized as the following. We first give the assumptions of the analytical model in Section IIA, followed by the description of the iterative algorithm in Section II-B. We then elaborate on the queueing subsystem in Section II-C and on the service subsystem in Section II-D, respectively. Finally, in Section II-E we will discuss how to achieve the throughput and delay performance.

\section{A. Assumptions}

To facilitate our discussion, we make the following assumptions:

- There are $N$ identical nodes in the network.

- At each node, packet arrivals are Poisson with the same rate $\lambda$ (packets/sec).

- The size of the packets (in bytes) from the upper layer is a random variable with probability distribution $f(n)$, where $f(n)=0$ for $n<N_{\min }$ or $n>N_{\max }$. In other words, the length of any packet is bounded.

- The queue at the MAC layer can store up to $K$ packets, which do not include the packet in the transmission buffer.

- The MAC header and the data packet are transmitted with rate $R_{d}$ (in bits/second), while RTS, CTS, ACK packets and the preambles are transmitted with rate $R_{c}$ (in bits/second).

- A packet transmission error occurs if any one bit in the packet is not correctly received. Moreover, every bit within the transmitted data packets encounters error with a fixed probability $\epsilon$. In other words, we assume that the channel is binary symmetric channel (BSC) ${ }^{1}$.

- To simplify the discussion we assume that control packets and frame headers of data packets are error free.

- The probability that one transmission attempt of a packet fails, denoted as $p$, does not depend on the backoff stage of the node.

- The packet service time is an integer multiple of a preset time unit $\tau$ (in seconds). This integer has an upper bound $I_{\max }$ because a server only tries to send one packet for finite number of times and each time the attempt has a finite duration.

- To simplify the discussion, we assume that the propagation delay is negligible.

${ }^{1}$ It is important to note that here we define the channel from the MAC layer perspective, which can be different to the channel in the physical layer, where various channel models and channel coding schemes can be used.

\section{B. The Iterative Algorithm}

To calculate the performance metrics of IEEE 802.11 DCF, we apply an iterative algorithm:

- Step1: Initialize $p_{I}=0$, where $p_{I}$ denotes the probability that a node has no packet to transmit in one time slot ${ }^{2}$.

- Step 2: With $p_{I}$, calculate $p_{t}$ and $p$ according to the model for binary exponential backoff (which will be discussed in Section II-D.1), where $p_{t}$ denotes the probability that a node will transmit in one time slot.

- Step 3: Calculate $q_{i}$ through the transfer-function approach using $p_{t}$ and $p$ (which will be discussed in Section II-D.2), where $q_{i}$ denotes the steady state probability that the packet service time is $i \tau$.

- Step 4: Calculate $p_{k}^{d}$ based on the M/G/1/K model (which will be discussed in Section II-C), where $p_{k}^{d}$ denotes the steady state probability that there are $k$ packets left in the queue at the time instance just before a packet departures.

- Step 5: Calculate a new $p_{I}$ based on $p_{k}^{d}$, which will also be discussed in Section II-C.

- Step 6: Calculate the MAC layer throughput $S$ and delay $T$ as shown in Section II-E.

- Step 7: If $S$ and $T$ converge with the previous values, then stop the algorithm; otherwise go to Step 2 with the updated $p_{I}$.

It is important to note that, since all nodes are identical and the traffic rate to each node is the same, we only need to analyze a single queue. In the following subsections, we will discuss how to calculate all parameters listed above.

\section{The Queueing Subsystem}

Based on the assumptions in Section II-A, the queueing system can be modelled as $M / G / 1 / K$. Following [5], we let $\xi(t)(t \geq 0)$ be the state of the queueing system at time $t$. The state space of $\xi(t)$ can then be defined as

$$
S=\left\{I, A_{0}, A_{1}, A_{2}, \cdots, A_{K}\right\},
$$

where $A_{k}$ means that the server is busy and there are $k$ packets waiting in the queue; $I$ means the server is idle, or in other words, the queue and the transmission buffer are empty.

Let $\delta_{n}$ be the time instance of the $n$-th packet departure. We now consider the embedded Markov process $\xi_{n}$, where $\xi_{n}$ is the state of the queueing system just before $\delta_{n}$, which is

$$
\xi_{n}=\xi\left(\delta_{n}^{-}\right) .
$$

This embedded Markov chain has state space $S^{\prime}=S-I=$ $\left\{A_{0}, A_{1}, A_{2}, \cdots, A_{K}\right\}$. Let $p_{i j}$ be the steady-state transition probability from state $A_{i}$ to $A_{j}$ for $\forall i, j \in[0, K]$, i.e.:

$$
p_{i j}=\lim _{n \rightarrow \infty} \operatorname{Pr}\left[\xi_{n+1}=A_{j} \mid \xi_{n}=A_{i}\right] .
$$

To calculate $p_{i j}$, we can use the service time distribution and the packet arrival rate. Define $\alpha(k)$ as the probability that

\footnotetext{
${ }^{2}$ Here we follow [8] and partition the continuous time axis into slots, where two consecutive slots are delimited by the event of a value change in the backoff counter.
} 
$k$ packets arrived during one packet service time. Since the packet arrival is a Poisson process with rate $\lambda$, we have

$$
\alpha(k)=\sum_{i=0}^{I_{\max }} q_{i} \frac{(\lambda i \tau)^{k} e^{-\lambda i \tau}}{k !} .
$$

Consequently, $p_{i j}$ can be calculated as

$$
p_{i j}= \begin{cases}\alpha(j) & i=0, j<K \\ 1-\sum_{k=0}^{K-1} \alpha(k) & i=0, j=K \\ 0 & i>0, j<i-1 \\ \alpha(j-i+1) & i>0, j<K \\ 1-\sum_{k=0}^{K-1} \alpha[k-i+1] & i>0, j=K .\end{cases}
$$

Since $p_{k}^{d}(0 \leq k \leq K)$ is the steady-state probability that $\xi_{n}=A_{k}$, we can calculate all $p_{k}^{d}$ by solving the embedded Markov chain with all $p_{i j}$.

Based on the $\mathrm{M} / \mathrm{G} / 1 / \mathrm{K}$ model, we can also calculate $p_{I}$ through

$$
p_{I}=\frac{p_{0}^{d}}{\lambda T^{s}+p_{0}^{d}} .
$$

where $T^{s}$ denotes the average packet service time, which is

$$
T^{s}=\sum_{i=0}^{I_{\max }} q_{i} \times(i \tau)
$$

\section{The Service Subsystem}

In this subsection, we first analyze the binary exponential backoff scheme of the DCF protocol using the Markov modelling technique introduced in [8]; we then calculate the service time distribution by the transfer-function approach [4], [5].

1) Binary Exponential Backoff: Similar to [8], we can formulate a two-dimensional discrete time embedded Markov chain with state $\left\{s_{n}, b_{n}\right\}$, where $n$ is the index of a slot, $b_{n}$ is the value of the backoff counter in slot $n$, and $s_{n}$ is the index of the backoff stage in slot $n$. Let the steady state probability of state $\left\{s_{n}=m, b_{n}=i\right\}$ be

$$
b_{m, i}=\lim _{n \rightarrow \infty} \operatorname{Pr}\left[s_{n}=m, b_{n}=i\right] .
$$

Based on the Markov chain, a closed-form solution for all $b_{m, i}$ can be derived. Since a transmission is initiated in slot $n$ if and only if $b_{n}=0$, we can obtain the first relationship between $p_{t}$ and $p$ [4].

In addition to the relationship between $p$ and $p_{t}$ described above, we note that a successful packet delivery can only occur when there is neither collision nor bit error in a transmission attempt. Therefore, we can calculate $p$ through

$$
p=1-\left(1-p_{c}\right)\left(1-p_{e}\right),
$$

where $p_{c}$ is the collision probability in any slot and $p_{e}$ is the packet error probability, which can be calculated through Eq. (10) and Eq. (11), respectively.

$$
\begin{gathered}
p_{c}=1-\left[1-\left(1-p_{I}\right) p_{t}\right]^{N-1} . \\
p_{e}=1-\sum_{n=N \min }^{N \max } f(n)(1-\varepsilon)^{8 n} .
\end{gathered}
$$

With these two relationships, we can calculate $p$ and $p_{t}$ numerically, as illustrated in [4], [8].

2) Service Time Distribution: Let $Q_{z}$ be the probability generating function (PGF) of $q_{i}$, which is

$$
Q(z)=\sum_{i=0}^{I_{\max }} z^{i} \cdot q_{i} .
$$

Due to the simplicity of notation in the z-transform domain and the one-to-one correspondence between $Q(z)$ and $\left\{q_{i}\right\}$, we will discuss how to calculate $Q(z)$ instead of individual $q_{i}$.

Similar to [5], we let $X_{n}$ be the length of slot $n$ and let $X_{n}^{\prime}$ be the length of the time interval (within slot $n$ ) during which the server is busy. Note that for saturated condition $X_{n} \equiv X_{n}^{\prime}$, while for unsaturated cases $X_{n}^{\prime} \leq X_{n}$. We can then apply the transfer-function approach, in which the packet transmission process is characterized by a linear system, as shown in Fig. 1.

In Fig. 1, $C(z)$ denotes the PGF of $X_{n}^{\prime}$ given that a collision occurred when the current node transmitted a packet; $S(z)$ denotes the PGF of $X_{n}^{\prime}$ given that the current node has successfully transmitted a packet without collision; and $H(z)$ denotes the PGF of $X_{n}^{\prime}$ given that the server of current node is busy but not transmitting. To simplify the notation, in Fig. 1, we define $H_{m}(z)$ as

$$
H_{m}(z)=\frac{1}{W_{m}} \sum_{i=0}^{W_{m}-1} H^{i}(z), \quad 0 \leq m \leq M .
$$

From Fig. 1 we can derive the transfer function of the linear system, which is $Q(z)$, as Eq. 14 .

Using the same technique that we developed in [5], we can calculate $C(z), S(z)$, and $H(z)$.

\section{E. Throughput and Delay}

Based on the M/G/1/K model, we can calculate the throughput of one queue, denoted as $S$, through

$$
S=\frac{\lambda\left(1-p^{M+1}\right) \bar{P}}{\lambda T^{s}+p_{0}^{d}},
$$

where $M$ is the retransmission limit and $\bar{P}$ is the average packet length in bits, which can be calculated through

$$
\bar{P}=\sum_{n=N \min }^{N \max } 8 n f(n) .
$$

To calculate the average packet delay $T$ (including queueing delay and service delay), we can use

$$
T=\frac{1}{\lambda}\left[\sum_{k=1}^{K-1} k p_{k+1}^{d}+K\left(p_{0}^{d}-1\right)\right]+(K+1) T^{s} .
$$




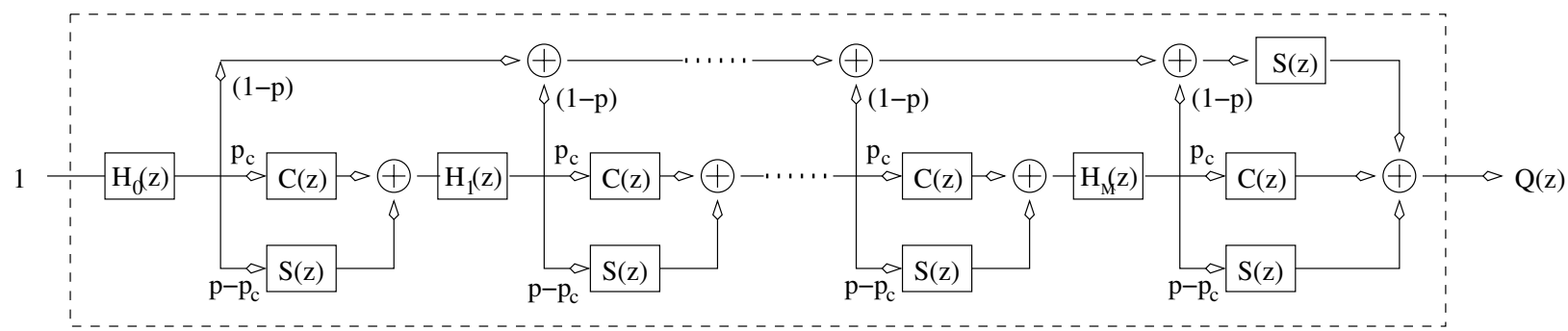

(a) $Q(z)$

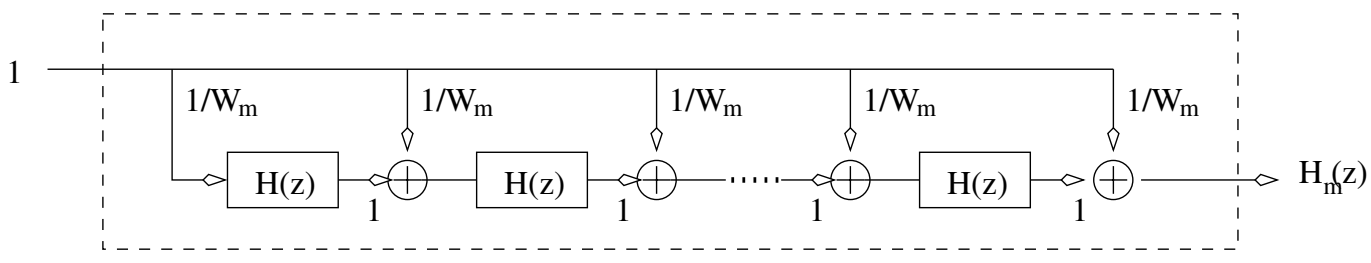

(b) $H_{m}(z)$

Fig. 1. Service system diagram.

$$
Q(z)=(1-p) S(z) \sum_{m=0}^{M}\left[\left[p_{c} C(z)+\left(p-p_{c}\right) S(z)\right]^{m} \prod_{i=0}^{m} H_{i}(z)\right]+\left[p_{c} C(z)+\left(p-p_{c}\right) S(z)\right]^{M+1} \times \prod_{i=0}^{M} H_{i}(z) .
$$

\section{Simulation AND Numerical Results}

In this section, we evaluate the performance of IEEE 802.11 DCF under different traffic and packet transmission error conditions through simulation and analytical results.

In our experiments, we use the default setting for IEEE $802.11 \mathrm{~b}$. In addition, we assume that all nodes in the network are located in a small area so that the propagation delay can be ignored. In our experiments, we let the packet arrivals to any node be a Poisson process with the same rate $\lambda$ (packets/s). Consequently, the total incoming traffic data rate is $R_{i}=N \bar{P} \lambda$ (bits/s). We further define the total incoming traffic load as $\rho=R_{i} / R_{d}$. Unless otherwise specified, we assume that the size of all packet are fixed to 1000 bytes. Due to limited space, we also let $N=10$ in this paper. For the analytical model, we let the time unit $\tau$ be $10 \mathrm{~ms}$ and let $I_{\max }$ be 60000 .

Fig. 2 first compares the simulation and analytical results of the service time distribution of IEEE 802.11 DCF, where we let $R_{d}=11 \mathrm{Mb} / \mathrm{s}, \epsilon=0$, and $\rho=1$ Erlang. We can observe that the results from our analytical model have a good match with the simulation results, which validates the sampling technique we utilize in the analytical model.

Fig. 3 shows the throughput versus traffic load of DCF with different bit error ratios (BERs), where the throughput of the analysis is $N \times S$. It can be observed that, our analytical model can accurately predict the throughput performance of DCF under different traffic and transmission error conditions. From Fig. 3 we can see a common trend for all transmission error conditions: if the traffic load is small, the overall throughput of DCF will be equal to the increase of incoming traffic data rates; and if the traffic load is higher enough, the throughput will become saturated. We can further observe from Fig. 3

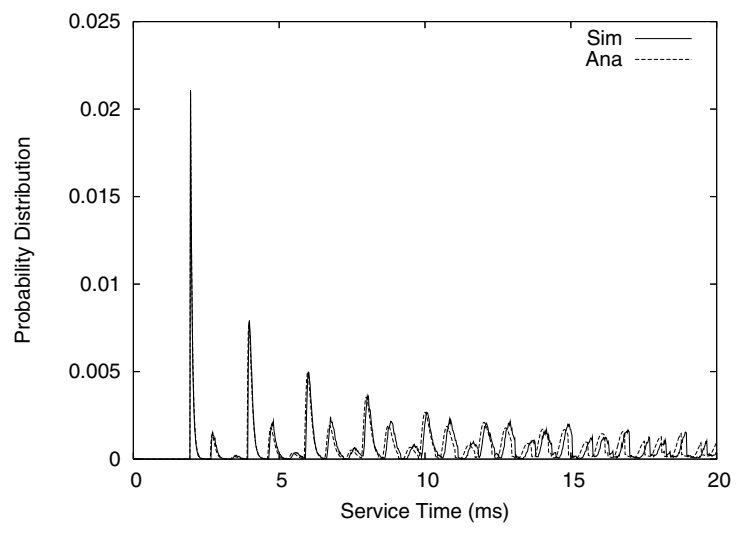

Fig. 2. Service time distribution $\left(R_{d}=11 \mathrm{Mb} / \mathrm{s}, \epsilon=0\right.$, and $\rho=1$ Erlang).

that a larger BER will lead to smaller saturated throughput. Particularly, in Fig. 3 (a), the throughput for $\rho=1$ Erlang is about $1.41 \mathrm{Mb} / \mathrm{s}$ for $\epsilon=10^{-6}$, and is about $1.32 \mathrm{Mb} / \mathrm{s}$ for $\epsilon=10^{-5}$. Another interesting observation is that, the normalized throughput, defined as $\left(N \times S / R_{d}\right)$, decreases with the increase of $R_{d}$. This phenomenon is primarily because the control overhead becomes larger with the increase of $R_{d}$.

In Fig. 3, we have discovered that the normalized throughput will be decreased if the channel data rate is higher. To overcome this problem, a possible approach is to increase the average length of incoming packets. However, as the average size of packets increases, the probability that a certain transmission is failed due to bit errors also increases, which will lead to a degradation of throughput performance. Intuitively, 


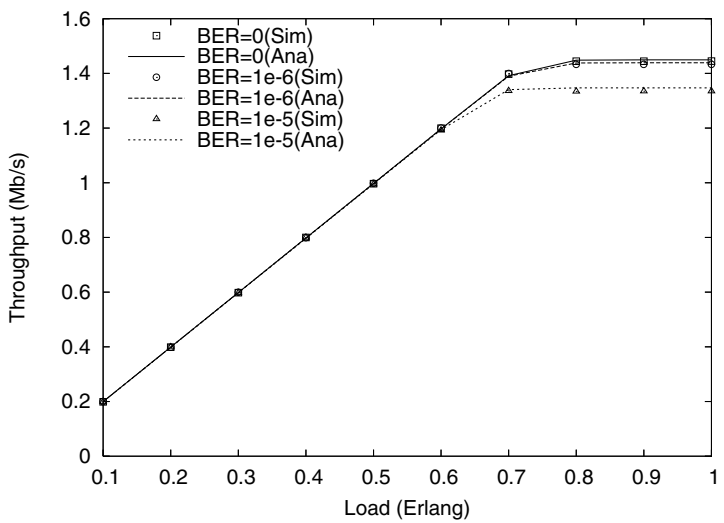

(a) $R_{d}=2 \mathrm{Mb} / \mathrm{s}$

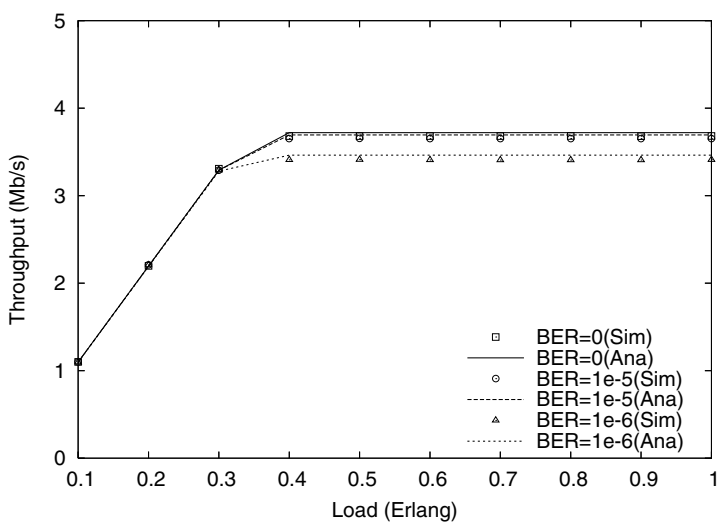

(b) $R_{d}=11 \mathrm{Mb} / \mathrm{s}$

Fig. 3. Through vs. load.

there may exist an optimal packet size which can result in the maximum throughput. This intuition is confirmed in Fig. 4, where we let $\epsilon=10^{-5}, R_{d}=11 \mathrm{Mb} / \mathrm{s}$, and $\rho=1$ Erlang. We can observe from Fig. 4 that the maximum throughput can be achieved if the packet size is about 4000 Bytes.

Finally, in Fig. 5, we show the delay performance versus traffic load under different bit error conditions, where we apply the same setting as that of Fig. 3 (a). We can observe that, our model can also accurately predict the delay performance.

\section{CONCLUSION}

In this paper, we develop a new analytical model to evaluate the performance of IEEE 802.11 DCF in binary symmetric channels, in which packet delivery can be failed due to bit transmission errors. The main contribution of our study is that we consider the impact of different realistic factors together, including binary exponential backoff, various incoming traffic loads, queueing system at the MAC layer, and the bit transmission errors, which has never been addressed in a comprehensive manner before. Extensive simulation and analysis results show that our analytical model can accurately predict the delay and throughput performance of IEEE 802.11 DCF under different traffic and transmission error conditions.

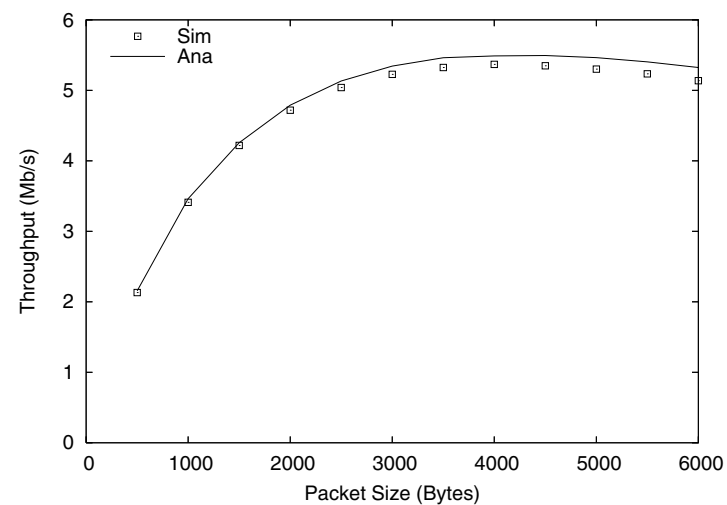

Fig. 4. Through vs. packet size ( $R_{d}=11 \mathrm{Mb} / \mathrm{s}, \epsilon=10^{-5}, \rho=1$ Erlang).

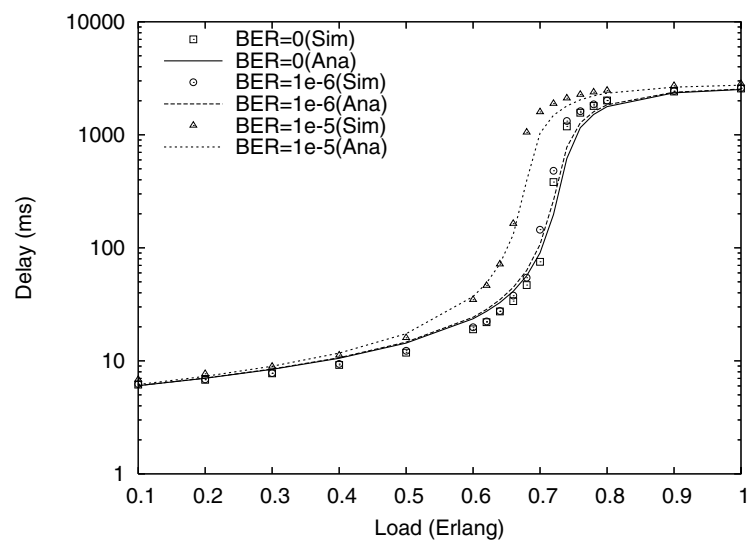

Fig. 5. Average delay vs. load $\left(R_{d}=2 \mathrm{Mb} / \mathrm{s}\right)$

\section{ACKNOWLEDGEMENT}

This work was supported in part by the US National Science Foundation under Faculty Early Career Development Award ANI-0093241.

\section{REFERENCES}

[1] Wireless LAN medium access control (MAC) and physical layer (PHY) specifications, ANSI/IEEE Std. 802.11, 1999, ISO/IEC 8802-11:1999(E).

[2] O. Tickoo and B. Sikdar, "Queueing analysis and delay mitigation in IEEE 802.11," in Proc. IEEE INFOCOM, Hong Kong, P. R. China, Apr. 2004.

[3] M. Ozdemir and A. B. McDonald, "A queuing theoretic model for IEEE 802.11 DCF using RTS/CTS," in IEEE Workshop on Local and Metropolitan Area Networks (LANMAN 2004), Apr. 2004, pp. 33-38.

[4] H. Zhai, Y. Kwon, and Y. Fang, "Performance analysis of IEEE 802.11 MAC protocol in wireless LAN," Wiley Journal of Wireless Communications and Moble Computing (WCMC), pp. 917-931, Dec. 2004.

[5] K. Lu, D. Wu, Y. Fang, and R. C. Qiu, "Performance analysis of a burstframe-based MAC protocol for ultra-wideband ad hoc networks," in Proc. IEEE ICC 2005, May 2005.

[6] Z. Hadzi-Velkov and B. Spasenovski, "Saturation throughput - delay analysis of IEEE 802.11 DCF in fading channel," in Proc. IEEE ICC, May 2003, pp. 121-126.

[7] Wireless LAN medium access control (MAC) and physical layer (PHY) specifications: Higher-speed physical layer extension in the $2.4 \mathrm{GHz}$ band, IEEE Std. 802.11b, 1999.

[8] G. Bianchi, "Performance analysis of the ieee 802.11 distributed coordination function," IEEE J. Select. Areas Commun., vol. 18, no. 3, pp. 535-547, Mar. 2000 